\title{
MicroRNA Expression Profile in Hyperoxia-Exposed Newborn Mice During the Development of Bronchopulmonary Dysplasia
}

\author{
Xiaoying Zhang MD, Wei Peng MM, Sheng Zhang MD, Chunzhi Wang MM, Xiyu He MM, \\ Zhimei Zhang MM, Lina Zhu MM, Yan Wang MD, Zhichun Feng MM
}

\begin{abstract}
BACKGROUND: Bronchopulmonary dysplasia (BPD) is a chronic lung disease of preterm neonates; the underlying pathogenesis is not fully understood. MicroRNAs (length 21-25 nucleotides) are ribonucleic acid (RNA) molecules that have important functions in development, cellular differentiation, apoptosis, proliferation, and migration; very little is known regarding their role in developmental lung diseases. METHODS: We exposed neonatal mice to either room air or $60 \%$ oxygen, beginning at birth, and we used microRNA microarray and real-time polymerase chain reaction on lung samples. RESULTS: The hyperoxia-exposed mice developed a lung injury that mimicked human BPD. Fifty-one microRNAs shared similar profiles in the hyperoxia-exposed BPD lungs and the normal lungs, which indicates that those microRNAs might play a protective role during the septation process. In the BPD lungs, compared to the control lungs, 14 microRNAs were up-regulated, and 7 microRNAs were down-regulated, which indicates that these microRNAs might play an important role in the development of BPD. Some of the candidate microRNAs can regulate cell proliferation. CONCLUSIONS: To our knowledge, this study is the first to identify microRNAs associated with BPD development, which provides a clue for further investigation of their function in BPD development. Key words: microRNA; bronchopulmonary dysplasia; hyperoxia; pathogenesis. [Respir Care 2011;56(7):1009-1015. (C) 2011 Daedalus Enterprises]
\end{abstract}

\section{Introduction}

Babies who are born prematurely or who experience respiratory problems shortly after birth are at risk for bronchopulmonary dysplasia (BPD), which is a common chronic

\footnotetext{
The authors are affiliated with the Department of Pediatrics, BaYi Children's Hospital of The General Military Hospital of Beijing People's Liberation Army, Beijing, People's Republic of China.
}

The authors have disclosed no conflicts of interest.

This study was partly supported by National Natural Science Foundation of China grant 81070524, 30973210 .

Supplementary materials related to this paper are available at http:// www.rcjournal.com.

Correspondence: Zhichun Feng MM, Department of Pediatrics, BaYi Children's Hospital of The General Military Hospital of Beijing People's Liberation Army, 5 Nanmencang Road, Dongcheng District, Beijing 100700, People's Republic of China. E-mail: fengzhichun81@ sina.com.

DOI: $10.4187 /$ respcare.01032 lung disease in preterm neonates and can have a multifactorial etiology, including hyperoxia exposure, premature lung, and neonatal therapies. ${ }^{1,2}$ Although the incidence and severity of respiratory distress syndrome have decreased with antenatal corticosteroid therapy and postnatal surfactant replacement, the overall incidence of BPD has been increasing. ${ }^{3,4}$ BPD is predominantly characterized by persistent abnormalities of lung structure and arrested lung development, including impaired alveolar and vascular growth. ${ }^{1,5}$ The pathogenesis of BPD is not fully understood.

MicroRNAs are 21-25 nucleotides long, non-coding RNAs that are involved in various biological processes, including cell proliferation, cell death, stress resistance, and tumorigenesis. ${ }^{6-8}$ Recent studies indicate that microRNAs are important in lung development ${ }^{9}$ and that abnormal microRNA levels cause aberrant expression of gene products that may contribute to lung disease, including lung cancers and asthma, ${ }^{10,11}$ so microRNAs might be useful diagnositic and/or prognostic markers for some diseases. To date, however, to our knowledge there have been no studies on microRNAs in the development of BPD. 
To assess whether microRNAs are involved in the development of BPD, we assayed microRNAs from $60 \%$ hyperoxia-exposed newborn mice-an ideal model because the hyperoxia-induced changes in lung structure closely mimic the histology of the altered lung architecture in human BPD. ${ }^{12-14}$ One critical determinant of normal postnatal lung architecture is septation, so we assayed the lungtissue microRNAs before, during, and after septation, on postnatal days 2,7 , and 21 .

\section{Methods}

\section{Animals}

We purchased newborn Kunming mice from the Chinese Academy of Sciences (Beijing, China) and randomly assigned 15 to room air and 15 to hyperoxia $\left(\mathrm{F}_{\mathrm{IO}_{2}} 60 \%\right.$ for 21 days), beginning at birth. Nursing dams were alternated every 24 hours between the air and hyperoxia litters. ${ }^{14}$ All the mice were maintained in a pathogen-free facility and used in accordance with our institutional guidelines for animal care. The animals were euthanized with intraperitoneal sodium pentobarbital after exposure on day 2, day 7 , or day 21 .

\section{Cells}

We cultured A549 cells in 1640 (Gibco-BRL, Invitrogen, Carlsbad, California), with $10 \%$ fetal bovine serum (Gibco-BRL). The cells were maintained in a humidified $37^{\circ} \mathrm{C}$ incubator, with an atmosphere of $5 \% \mathrm{CO}_{2}$.

\section{Histology}

To prepare the lungs for histology we opened the chest cavity and exposed the trachea, inserted a blunt cannula and tied it to the trachea, and inflated the lungs with phosphate buffered saline $4 \%$ formalin fixative at $25 \mathrm{~cm} \mathrm{H}_{2} \mathrm{O}$. After overnight fixation at $4{ }^{\circ} \mathrm{C}$, the tissue was dehydrated and paraffin embedded, cut into 5- $\mu \mathrm{m}$ sections, stained with hemotoxylin and eosin, and examined with light microscopy. ${ }^{15,16}$ We performed radial alveolar counts, as previously described. ${ }^{17}$ From the center of the respiratory bronchiole, a perpendicular line is drawn to the edge of the acinus, and the number of the septa intersected by that line are counted. The radial alveolar count was made at magnification $\times 100$.

\section{Lung RNA Assay}

Total RNA was isolated from individual whole lungs with an microRNA isolation kit (miRVana, Ambion, Austin, Texas) per the manufacturer's instructions. RNA quality was controlled with an optical density (OD) 260/280 ratio of 1.8 to 2 and an OD 260/230 of $\geq 1.8$, and $1 \mu \mathrm{g}$ total RNA was used.

\section{Microarray Methods}

We pooled 3 individual total RNA samples from the same time-point as one sample, to minimize biological variability and the number of microarrays to generate the necessary data. These RNA samples were treated with poly(A) tailing, flash tag ligation, and hybridization (GeneChip miRNA Array, Affymetrix, Santa Clara, California) per the manufacturer's protocol.

\section{Microarray Data Analysis}

We scanned the chips, computed the signal intensity (GeneChip Command Console 1.1 software, Affymetrix, Santa Clara, California), and summarized, normalized, conducted quality-control procedures on the data (microRNA QC Tool software, Affymetrix, Santa Clara, California), and created cluster and tree-view graphs, as previously described. ${ }^{18}$

\section{Reverse Transcription Reaction and Quantitative Real-Time Polymerase Chain Reaction}

We purified the total RNAs (Absolutely RNA Nanoprep kit, Stratagene, Agilent Technologies, Santa Clara, California). We carried out reverse transcription reactions and real-time-polymerase chain reaction as previously described. ${ }^{16}$ We calculated the relative expression of microRNA compared to $u 6$ with the $2^{-\Delta \Delta \mathrm{Ct}}$ method. ${ }^{16}$ (See the supplementary materials related to this paper at http:// www.rcjournal.com.)

\section{Transient Transfection}

Transfections were performed (Lipofectamine 2000, Invitrogen, Carlsbad, California) per the manufacturer's instructions and our previous report. ${ }^{16}$ Cells $\left(1-3 \times 10^{6}\right)$ grown to a confluency of $50-60 \%$ in $10-\mathrm{cm}$ petri dishes were transfected with different double-stranded microRNA mimics (600 pmol, GenePharma, Shanghai, People's Republic of China) or microRNA mock, and the cells were harvested 48 hours post-transfection.

\section{Measurement of Cell Proliferation}

A549 cells transfected with microRNAs $\left(1 \times 10^{4}\right)$ were plated in 96-well plates, and the cell proliferation was measured with a proliferation kit (XTT II, Boehringer, Mannheim, Germany). ${ }^{16}$ Optical density was read with a microplate reader (Bio-Rad, Hercules, California). 


\section{Statistical Analysis}

Data are presented as mean $\pm \mathrm{SD}$. We used analysis of variance to calculate differences between body weight, radial alveolar count, and proliferation, and if $P$ was $<.05$, we performed the least significant difference test. We used the Pearson test to determine the correlation of the microRNA levels between the microRNA microarray assay and the real-time polymerase chain reaction assay. Differences were deemed statistically significant at $P<.05$.

\section{Results}

\section{Effects of 60\% Oxygen Exposure on Newborn Mice}

There was no mortality in either group, but the hyperoxia group had significantly lower body weight on day 7 $(8.10 \pm 0.26 \mathrm{~g}$ vs $8.94 \pm 0.24 \mathrm{~g}, P=.01)$ and on day 21 (21.04 $\pm 0.26 \mathrm{~g}$ vs $26.7 \pm 0.31 \mathrm{~g}, P=.001)$, but not on day $2(5.73 \pm 0.25 \mathrm{~g}$ vs $6.09 \pm 0.23 \mathrm{~g}, P=.14)$. Structural changes were evident in the hyperoxia and control mice lungs on day 2, day 7, and day 21 (ie, before, during, and after septation). At 2 days the hyperoxia mice had no obvious qualitative differences in the lung regions characterized by either large distal air spaces or absence of interstitial thickening. However, at 7 days the hyperoxia mice had heterogeneous lung structure changes, including patchy areas of parenchymal thickening, small air spaces interspersed in enlarged air spaces. At 21 days the hypoxia mice had a lung injury pattern that has morphologic similarities to human BPD (Fig. 1A). Hyperoxia reduced radial alveolar count by $34 \%$ on day $7(P=.001)$ and by $33 \%$ on day $21(P=.001)$ (see Fig. 1B). The alveolar enlargement and decrease in surface area are associated with decreased oxygen transfer and arterial oxygen saturation, resulting in the characteristic BPD-related reduction in lung function.

\section{MicroRNA Expression Differences During Septation}

To determine the microRNA quantities during the septation process, we used a novel microRNA array-based approach. We report these data as fold-change values, and we considered $\mathrm{a} \geq 2$-fold change of expression important and selected those changes for study. We randomly selected 6 microRNAs that exhibited $\geq 2$-fold change in expression to validate the data with real-time polymerase chain reaction for mature microRNAs (see the supplementary materials related to this paper at http://www.rcjournal. com). The correlation coefficient between microarray and real-time polymerase chain reaction for the 6 microRNAs was $0.884(P=.007)$, which means the microarray data agreed with the real-time polymerase chain reaction data and the 2 methods had very high internal consistency. We

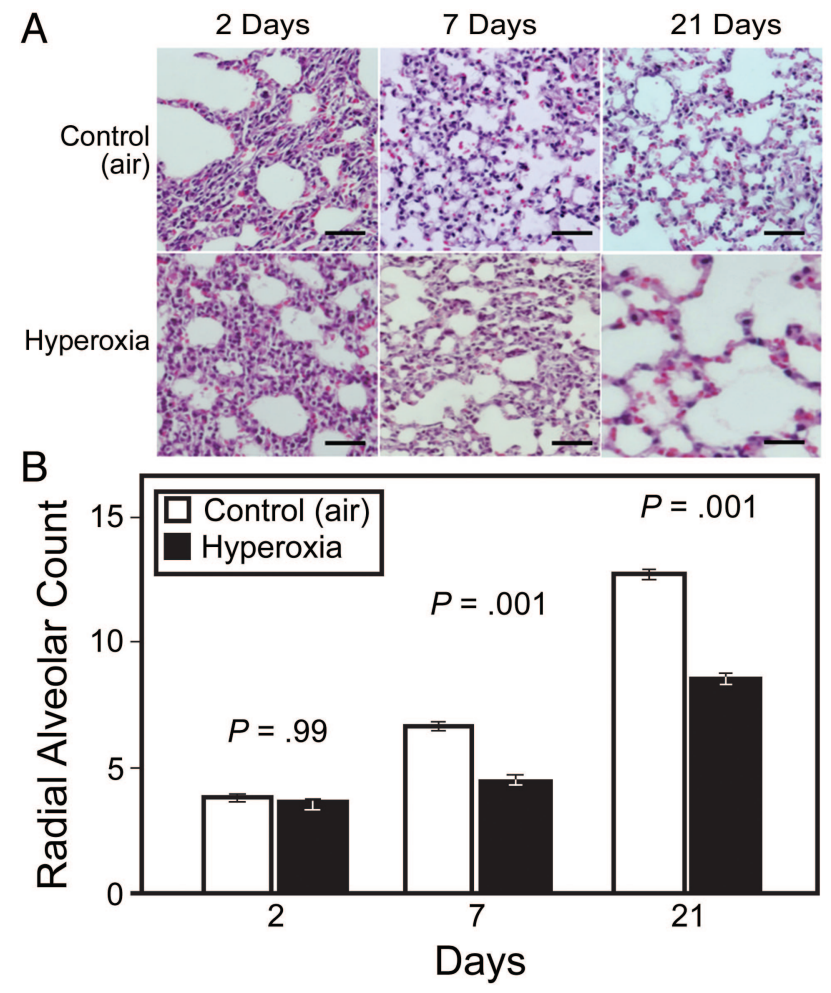

Fig. 1. Lung development at 2 days, 7 days, and 21 days after birth in mice raised in a hyperoxic environment (60\% oxygen) versus control mice (normal air environment). A: Lung development is markedly abnormal (enlarged air spaces and simplified structure) in the hyperoxia group on day 7 and day 21 , compared to the control group. The black bars represent $100 \mu \mathrm{m}$. B: The radial alveolar count is significantly different on day 7 and day 21 . The whisker bars indicate standard deviations.

therefore used microarray for analysis of the microRNAs profiles. We determined the expression changes of individual microRNAs via cluster analysis. During the septation process, 72 microRNAs changed expression level in the control group (Fig. 2A) and 87 microRNAs changed in the hyperoxia group (see Fig. 2B). Among those microRNAs, 51 were shared by the control and hyperoxia groups, and the expression levels (fold-changes) were very similar (see the supplementary materials related to this paper at http://www.rcjournal.com). Of note, miR-29a was the most highly up-regulated microRNA, and miR-100, miR-3425p, miR-497 and the miR-30 family were also highly upregulated in both groups. MicroRNAs greatly down-regulated in both groups were miR-122, miR-298, miR-411, miR-541, miR-382, miR-134, miR-668, miR-485, miR-409, miR-329, miR-665, miR-433, miR-127 and miR-379. These data show similarities during septation in the 2 groups, which indicates that these microRNAs play an important role during septation, whatever the hyperoxia injury. 

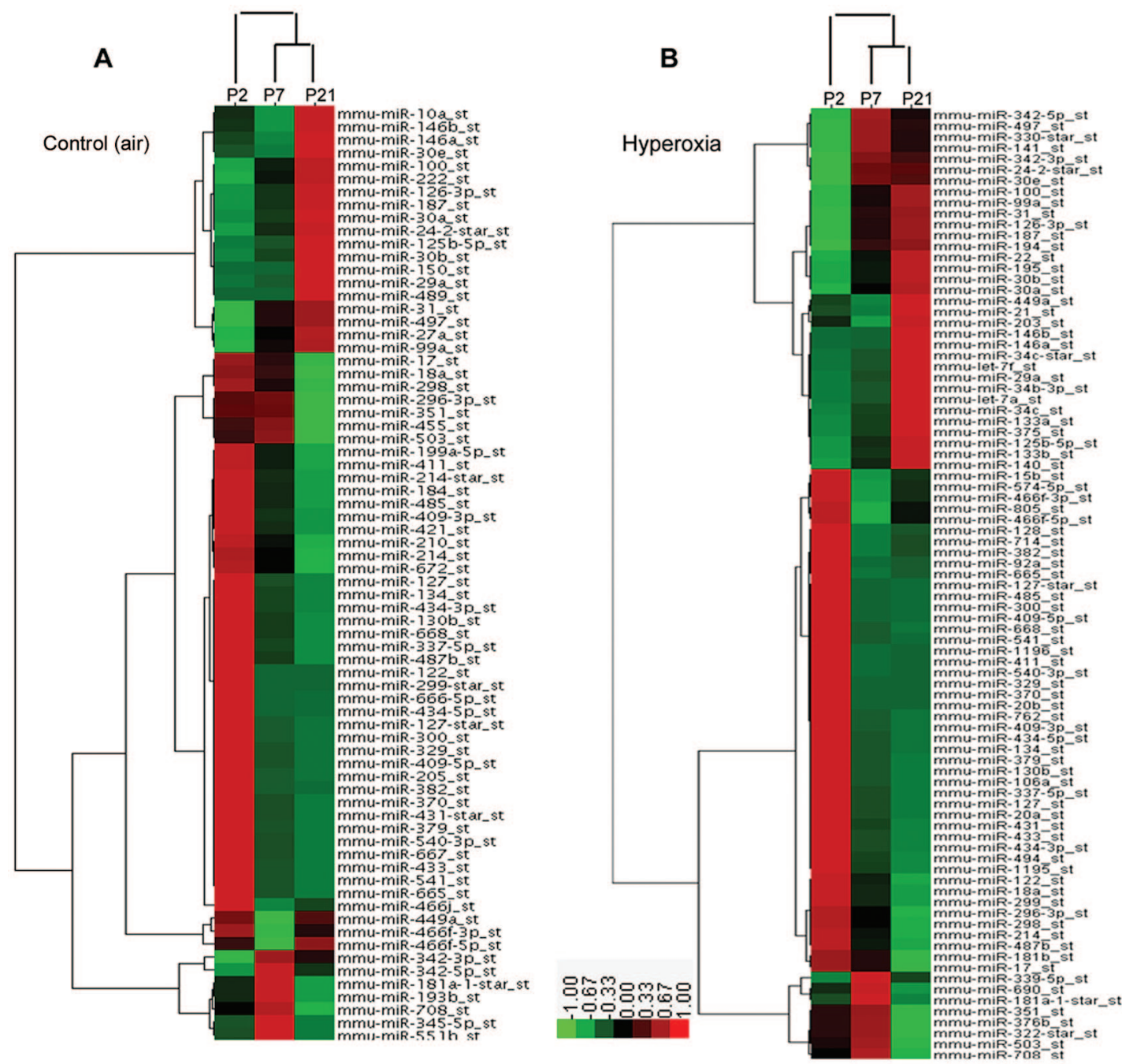

Fig. 2. MicroRNA expression in neonatal mice lung on days 2 (P2), 7 (P7), and 21 (P21). A: Control group. B: Hyperoxia group. The experimental model was hierarchically clustered in both dimensions and plotted on a heat map. Each small block indicates its own expression level. Red denotes a high expression level, green denotes a low expression level, and gray denotes no detectable signal. The whisker bars indicate standard deviations.

\section{MicroRNA Expression Differences During BPD Development}

Detailed account of the microRNA expression profiling during BPD development has not previously been reported, so we compared the groups' expression profiles (Figs. 3 and 4). Twenty-one microRNAs levels were greatly changed in the control mice lungs, but there were no substantial changes in the BPD mice lungs (see Fig. 3A), whereas 36 microRNAs levels showed aberrant regulation in the BPD mice lungs, but no substantial changes in the control mice lungs (Fig. 3B), which implies these microRNAs might be important in BPD development. Furthermore, these microRNA levels on day 7 were often intermediate between those on day 2 and day 21 , which suggests a gradual developmental shift in the expression pattern. In particular, on day 2 the levels of miR-20b, miR-106a, miR-128, miR-883b-3p, and miR-15b (see Fig. 4A); on day 7 the levels of miR-122, miR-30e, and miR-365-5p (Fig. 4B); and on day 21 the levels of miR-133a, miR-205, miR-379, miR-449a, miR-431, and let-7f (see Fig. 4C) were more than 2-fold higher in the BPD mice than in the control mice. Conversely, on day 2 the levels of miR-299, miR-139p-3p, miR-300 star, and miR-122; on day 7 the levels of miR-335p-5p and miR-714; and on day 21 the level of miR-720 were more than 2-fold lower in the BPD mice (Fig. 4A-C).

\section{MicroRNA Can Regulate Cell Proliferation}

Next we studied whether the candidate microRNAs affect cell biology. We analyzed the proliferation potential 
A

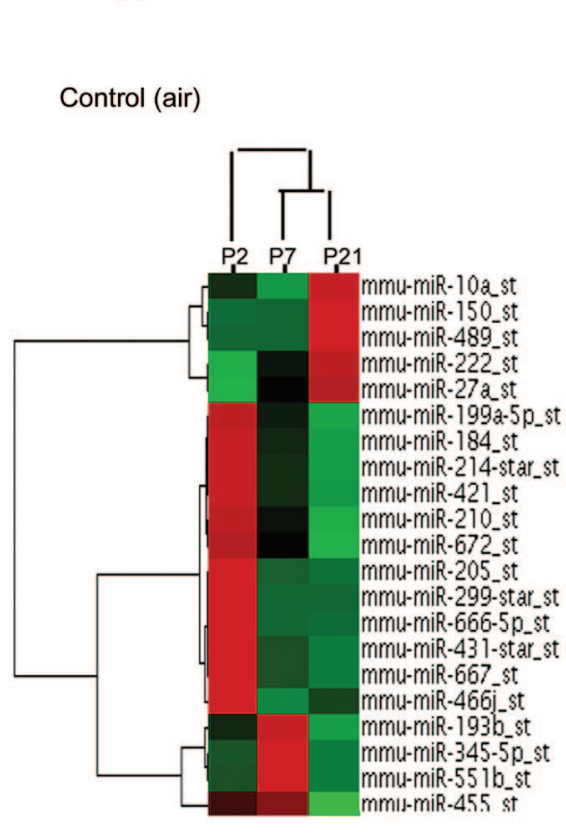

B<smiles></smiles>
mmu-miR-194_st mmu-miR-140_st mmu-miR-195_st mmu-miR-22_st mmu-let-7a_st mmu-miR-34b-3p_st mmu-let-7f_st mmu-let-7f_st
mmu-miR-34c-star_st mmu-miR-133ast mmu-miR-375_st mmu-miR-34c mmumiR-2 mmu-miR-203_st mmu-miR-21_st mmu-miR-14i_st mmu-miR-330-star_st mmu-miR-339-5p_st mmu-miR-322-star_st mmu-miR-376b_st mmu-miR-690_st mmu-miR-181b_st mmu-miR-1196_st mmu-miR-20b_st mmu-miR-92ast mmu-miR-128 st mmu-miR-714_st mmu-miR-106a_st mmu-miR-762_st mmu-miR-1195 st mmu-miR-494_st mmu-miR-20àst mmu-miR-431_st mmu-miR-299_st mmu-miR-15b_st mmu-miR-574-5p_st mmu-miR-805 st

Fig. 3. Differential MicroRNA expression in neonatal mice lung on days 2 (P2), 7 (P7), and 21 (P21). A: Control group. B: Hyperoxia group. The experimental model was hierarchically clustered in both dimensions and plotted on a heat map. Each block indicates its own expression level. Red denotes a high expression level, green denotes a low expression level, and gray denotes no detectable signal. Twenty-one microRNA expression levels were greatly dysregulated, compared to the air-exposed control mice lungs. B: Thirty-six microRNAs levels showed aberrant regulation in the hyperoxia-exposed BPD mice lungs.

of cells (A549) transfected with microRNAs (hsa-let-7f, hsa-miR-29a, hsa-miR-21, hsa-miR-20b, hsa-miR-127, and hsa-miR-134) or miR-mock. The results revealed that overexpression of miR-21 or $-20 \mathrm{~b}$ induced cell proliferation significantly $(P=.02$ and $P=.007$, respectively), whereas miR-29a, $-127,-134$, and let-7f significantly reduced cell proliferation $(P=.003, P=.03, P=.006$ and $P=.006$, respectively), compared to miR-mock (Fig. 5). Overexpression of these microRNAs significantly affected cell proliferation in vitro, which indicates that these microRNAs are important in the biological and pathological processes.

\section{Discussion}

Little is known about the expression profiles and roles of microRNAs in BPD development, so we compared microRNA expression in control and hyperoxia-exposed mice and investigated their role during septation. The mouse is an ideal animal to study BPD development, because mouse lung displays morphological and pathological BPD characteristics during the procedure of branching morphogenesis, vascularization, and alveolarization. ${ }^{12-14}$ On day 21 the mice lungs displayed the characteristic BPD-related reduction in lung function. We predicted that some microRNAs play an important protective role during septation, as there were some similarities between the control and BPD lungs from day 2 to day 21. In both groups, miR-29a and the miR-30 family were the most highly up-regulated microRNAs, which agrees with the report by Williams et al. ${ }^{19}$ However, 14 microRNAs (miR-20b, miR106a, miR-128, miR-883b-3p, miR-15b, miR-122, miR-30e, miR-365-5p, miR-133a, miR-205, miR-379, miR-449a, miR-431, and miR-431) were greatly up-regulated in the BPD lungs, compared to the control lungs, whereas 7 microRNAs (miR-299, miR-139p-3p, miR300 star, miR-122, miR-335p-5p, miR-714, and miR-720) were significantly down-regulated in the BPD lungs, compared to the control lungs, some of which could regulate cell proliferation. All together, these results suggest that these microRNAs are coordinately expressed and central to BPD development.

Multiple microRNAs were differentially expressed during BPD development. Although the functional role of these changes in microRNA expression is unknown, ex- 

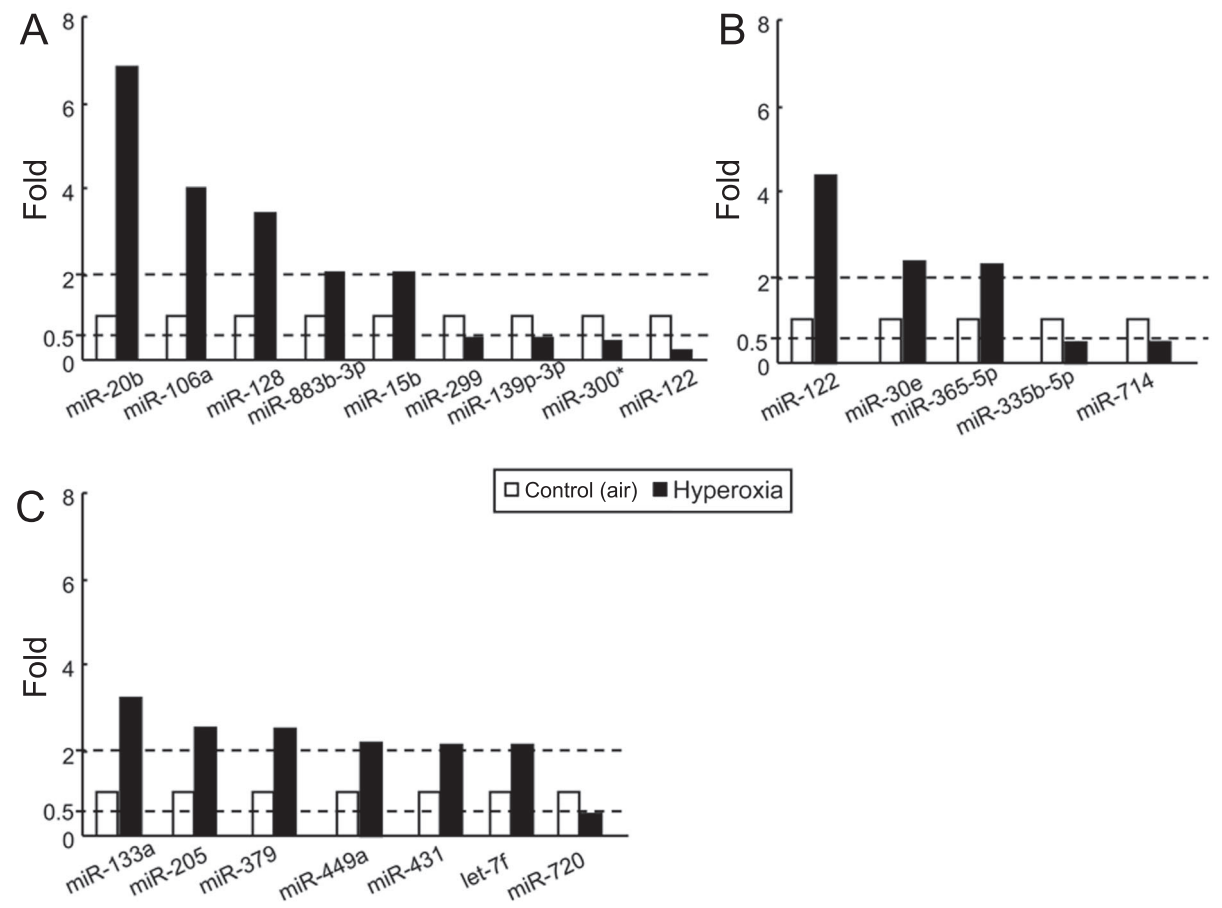

Fig. 4. Differential expression of microRNAs in a control group and a hyperoxia group of neonatal mice. A: Day 2. B: Day 7. C: Day 21. The dotted lines represent a 2 -fold change in expression.

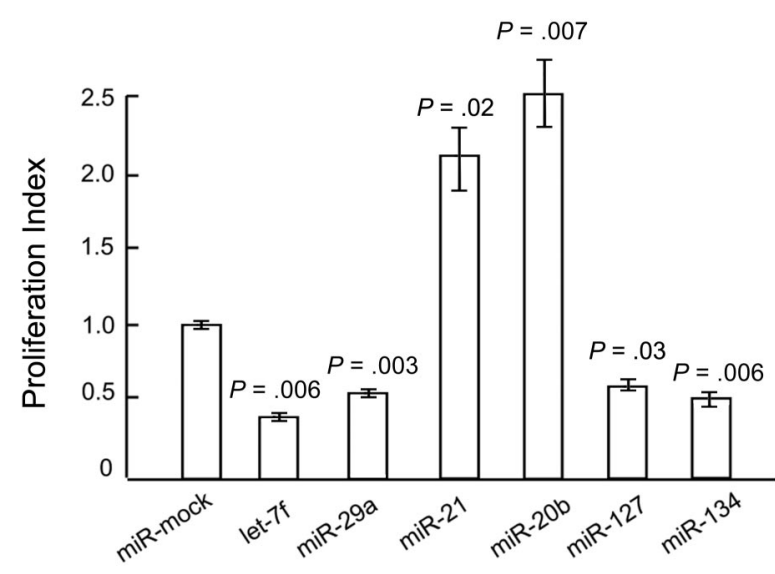

Fig. 5. Candidate microRNAs that significantly affected cell proliferation, as assessed via XTT (2-3-bis(2-methoxy-4-nitro-5-sulfophenyl)-5-[(phenylamino) carbonyl]-2H-tetrazolium hydroxide) 48 hours after transfection, compared to miR-mock. The whisker bars indicate standard deviations.

amination of their genomic localization shows that 15 of the microRNAs down-regulated during BPD process in the septation developing lungs (miR-541, miR-382, miR134, miR-668, miR-485, miR-409, miR-411, miR-299, miR-494, miR-431, miR-329, miR-665, miR-433, miR127 and miR-379) map to the $12 \mathrm{~F} 1$ in mice, and are contained within 3 separate clusters. miR-20b and miR-106a, a microRNA family, were up-regulated in the BPD lungs, compared to the control lungs, on day 2 , and are located at mouse chromosome XA5. However, the miR-30 family (miR-30a, miR-30b, and miR-30e) is located on other mouse chromosomes. These microRNAs might be coordinately expressed, because they are encoded by the same pri-miR or pre-miR precursor or because they are driven by the same transcription factor or regulated by the same cellular mechanism(s) and play a key role in the septation processes and BPD development.

Although these microRNAs were previously shown to be associated with cell differentiation, biological metabolism, and cancer regulation, no information has been available on the role of specific microRNAs in BPD development. For example, miR-29a is reported as a potentially negative regulator of lung cancer ${ }^{20}$; the microRNA gene maps to mouse chromosome $6 \mathrm{~A} 3$, a fragile site that is associated with several cancers. In our study, miR-29a was up-regulated in the hyperoxia group, compared to the control group, and it expressed at a higher level in developing lungs in both groups. miR-29a also reduced the proliferation of A549, which indicates an important role in the regulation of cell biology. Importantly, miR-133a regulates cardiomyocyte proliferation and suppresses smooth muscle gene expression in the heart. ${ }^{21}$ Chiba et al found that miR-133a contributed to up-regulation of RhoA in bronchial smooth muscle cells, which was one of the causes of bronchial hyper-responsiveness. ${ }^{22} \mathrm{We}$ found that miR- 
133a was up-regulated in the BPD lungs from day 2 to day 21 , but not in the control lungs, and on day 21 , miR133a was greatly up-regulated in the BPD lungs, compared to the control lungs, which suggests that miR-133a is important in BPD development as the lung matures. And epigenetic regulation of miR-370 by interleukin- 6 contributes to tumor growth. ${ }^{23}$ However, overexpression of let-7 in the human lung epithelial A549 cell line inhibits cell growth, ${ }^{24}$ which is consistent with our results. Interestingly, miR-20b and let-7f were all up-regulated in the BPD lungs, compared to the control lungs, but they had different effects on cell proliferation. Overexpression of miR$20 \mathrm{~b}$ significantly increased cell proliferation, whereas let- $7 \mathrm{f}$ significantly reduced cell proliferation. However, we do not know whether the different effects are caused by the different genera (Homo sapiens vs Mus) or the different BPD stages (day 2 vs day 21), or what is the critical mechanism that initiates and advances BPD mediated by microRNAs. This report provides a preliminary understanding of the roles of microRNAs in BPD development.

\section{Conclusions}

Fifty-one microRNAs share similar profiles in the BPD lungs and control lungs. Fourteen microRNAs were up-regulated and 7 were down-regulated in the BPD lungs, compared to the control lungs. Some of the candidate microRNAs can affect cell regulation. This is the first study to identify the microRNAs associated with BPD development. The exact functions of these microRNA genes remains to be determined, but we believe this study provides a basis for further investigation of their function in BPD development.

\section{REFERENCES}

1. Baker CD, Ryan SL, Ingram DA, Seedorf GJ, Abman SH, Balasubramaniam V. Endothelial colony-forming cells from preterm infants are increased and more susceptible to hyperoxia. Am J Respir Crit Care Med 2009;180(5):454-461.

2. Deakins KM. Bronchopulmonary dysplasia. Respir Care 2009;54(9): 1252-1262.

3. Kwinta P, Grudzien A, Pawlik D, Olechowski W, Lauterbach R, Pietrzyk JJ. [Prevalence and risk factors of bronchopulmonary dysplasia among extremely low birth weight newborns of regional birth cohort of south-east Poland]. Przegl Lek 2009;66(1-2):14-20. Article in Polish.

4. Woynarowska M, Rutkowska M, Szamotulska K. [Risk factors, frequency and severity of bronchopulmonary dysplasia (BPD) diagnosed according to the new disease definition in preterm neonates]. Med Wieku Rozwoj 2008;12(4 Pt 1):933-941. Article in Polish.

5. Balasubramaniam V, Mervis CF, Maxey AM, Markham NE, Abman $\mathrm{SH}$. Hyperoxia reduces bone marrow, circulating, and lung endothelial progenitor cells in the developing lung: implications for the pathogenesis of bronchopulmonary dysplasia. Am J Physiol Lung Cell Mol Physiol 2007;292(5):L1073-1084.

6. Cheng J, Zhou L, Xie QF, Markham NE, Abman SH, Xing CY, et al. The impact of miR-34a on protein output in hepatocellular carcinoma HepG2 cells. Proteomics 2010;10(8):1557-1572.
7. Hu W, Chan CS, Wu R, Zhang C, Sun Y, Song JS, et al. Negative regulation of tumor suppressor p53 by microRNA miR-504. Mol Cell 2010;38(5):689-699.

8. Gao P, Bai X, Yang L, Lv D, Li Y, Cai H, et al. Over-expression of osa-MIR396c decreases salt and alkali stress tolerance. Planta 2010; 231(5):991-1001.

9. Williams AE, Perry MM, Moschos SA, Lindsay MA. microRNA expression in the aging mouse lung. BMC Genomics 2007;8(172.

10. Du L, Pertsemlidis A. microRNAs and lung cancer: tumors and 22-mers. Cancer Metastasis Rev 2010;29(1):109-122.

11. Mohamed JS, Lopez MA, Boriek AM. Mechanical stretch upregulates microRNA-26a and induces human airway smooth muscle hypertrophy by suppressing glycogen synthase kinase-3\{beta\}. J Biol Chem 2010;285(38):29336-29347.

12. Yi M, Jankov RP, Belcastro R, Humes D, Copland I, Shek S, et al. Opposing effects of $60 \%$ oxygen and neutrophil influx on alveologenesis in the neonatal rat. Am J Respir Crit Care Med 2004;170(11): 1188-1196.

13. Jankov RP, Luo X, Belcastro R, Copland I, Frndova H, Lye SJ, et al. Gadolinium chloride inhibits pulmonary macrophage influx and prevents $\mathrm{O}(2)$-induced pulmonary hypertension in the neonatal rat. Pediatr Res 2001;50(2):172-183.

14. Ahmed MN, Suliman HB, Folz RJ, Nozik-Grayck E, Golson ML, Mason SN, et al. Extracellular superoxide dismutase protects lung development in hyperoxia-exposed newborn mice. Am J Respir Crit Care Med 2003;167(3):400-405.

15. Bry K, Whitsett JA, Lappalainen U. IL-1beta disrupts postnatal lung morphogenesis in the mouse. Am J Respir Cell Mol Biol 2007;36(1): $32-42$.

16. Zhang X, Liu S, Hu T, Liu S, He Y, Sun S. Up-regulated microRNA143 transcribed by nuclear factor kappa B enhances hepatocarcinoma metastasis by repressing fibronectin expression. Hepatology 2009;50(2):490-499.

17. Balasubramaniam V, Tang JR, Maxey A, Plopper CG, Abman SH. Mild hypoxia impairs alveolarization in the endothelial nitric oxide synthase-deficient mouse. Am J Physiol Lung Cell Mol Physiol 2003; 284(6):L964-L971.

18. Takamizawa J, Konishi H, Yanagisawa K, Tomida S, Osada H, Endoh $\mathrm{H}$, et al. Reduced expression of the let-7 microRNAs in human lung cancers in association with shortened postoperative survival. Cancer Res 2004;64(11):3753-3756.

19. Williams AE, Moschos SA, Perry MM, Barnes PJ, Lindsay MA. Maternally imprinted microRNAs are differentially expressed during mouse and human lung development. Dev Dyn 2007;236(2):572580.

20. Fabbri M, Garzon R, Cimmino A, Liu Z, Zanesi N, Callegari E, et al. MicroRNA-29 family reverts aberrant methylation in lung cancer by targeting DNA methyltransferases 3A and 3B. Proc Natl Acad Sci USA 2007;104(40):15805-15810.

21. Liu N, Bezprozvannaya S, Williams AH, Qi X, Richardson JA, Bassel-Duby R, et al. MicroRNA-133a regulates cardiomyocyte proliferation and suppresses smooth muscle gene expression in the heart. Genes Dev 2008;22(23):3242-3254.

22. Chiba Y, Tanabe M, Goto K, Sakai H, Misawa M. Down-regulation of miR-133a contributes to up-regulation of Rhoa in bronchial smooth muscle cells. Am J Respir Crit Care Med 2009; 180(8):713-719.

23. Meng F, Wehbe-Janek H, Henson R, Smith H, Patel T. Epigenetic regulation of microRNA-370 by interleukin-6 in malignant human cholangiocytes. Oncogene 2008;27(3):378-386.

24. Johnson CD, Esquela-Kerscher A, Stefani G, Byrom M, Kelnar K, Ovcharenko D, et al. The let-7 microRNA represses cell proliferation pathways in human cells. Cancer Res 2007;67(16):7713-7722. 